\title{
EMERGÊNCIA DE HAEMATOBIA IRRITANS NAS MASSAS FECAIS DE BOVINOS DAS RAÇAS NELORE E PANTANEIRA NO PANTANAL MATO-GROSSENSE ${ }^{1}$
}

\author{
FABIANA TAVARES PIRES DE SOUZA SERENO² e JOSÉ ROBSON BEZERRA SERENO ${ }^{3}$
}

\begin{abstract}
RESUMO - Este trabalho foi realizado na Fazenda Nhumirim de propriedade da Embrapa-Centro de Pesquisa Agropecuária do Pantanal, em Corumbá, MS, no período de 11/93 a 05/94, com coletas mensais de massas fecais de bovinos das raças Nelore e Pantaneira, com o objetivo de comparar o número de moscas-do-chifre nas duas raças e determinar qual das duas exerce maior atração sobre a mosca-do-chifre (Haematobia irritans). De 112 fezes bovinas recolhidas de cada raça, observou-se que em Nelore, 107 (95,5\%) massas fecais apresentavam pupas de dípteros, e apenas 72 (64,3\%) tinham pupas de H. irritans; na raça Pantaneira, das 105 (94,0\%) massas fecais com pupas de dípteros apenas $60(53,6 \%)$ apresentavam $H$. irritans. Os resultados das medições comparativas dos parâmetros comprimento, largura, diâmetro, altura e volume entre as massas fecais demonstraram que não houve diferença significativa $(\mathrm{P}>0,05)$. O número de pupas de dípteros coletadas nas massas fecais de bovinos da raça Pantaneira $(\mathrm{n}=2892)$ foi maior do que em Nelore $(\mathrm{n}=1715)(\mathrm{P}<0,05)$. Em relação à H. irritans, observouse maior número de pupas nas massas fecais de bovinos da raça Pantaneira $(n=399)(P<0,05)$ do que em Nelore $(\mathrm{n}=237)$. As massas fecais dos Pantaneiros apresentavam também maior $(\mathrm{P}<0,05)$ número total de pupas por volume de fezes. Os resultados indicam que os bovinos da raça Pantaneira exercem maior atração sobre a mosca-do-chifre.
\end{abstract}

Termos para indexação: controle biológico, moscas.

\section{EMERGENCE OF HAEMATOBIA IRRITANS IN FECES OF NELORE AND PANTANEIRA BREED IN THE PANTANAL MATO-GROSSENSE, BRAZIL}

\begin{abstract}
This study was carried out at Nhumirim farm, property of Embrapa-Centro de Pesquisa Agropecuária do Pantanal, Corumbá, MS, Brazil, from 11/93 to 05/94, with manure samples of Nelore and Pantaneira breeds collected to compare the number of horn flies (Haematobia irritans) on manure samples of both bovine breeds. Out of the 112 bovine samples collected from each breed, 72 (64.3\%) Nelore manure samples showed pupa of H. irritans; in Pantaneira, out of 105 (94\%) manure samples with fly pupas, only $60(53.6 \%)$ exhibited $H$. irritans. The results of comparative measurements of length, width, height and volume parameters among the manure samples, showed no significant difference ( $\mathrm{t}$ test $-\mathrm{P}>0.05$ ) between Nelore and Pantaneira breeds. The number of fly pupas collected from the manure samples of Pantaneira breed $(\mathrm{n}=2892)$ was higher than in Nelore breed $(\mathrm{n}=1715)(\mathrm{P}<0.05)$. With relation to $H$. irritans, a higher number of pupas was observed in the manure samples of Pantaneira breed $(\mathrm{n}=399)(\mathrm{P}<0.05)$ than in Nelore breed $(\mathrm{n}=237)$. The manure samples of Pantaneira also presented a higher $(\mathrm{P}<0.05)$ total number of pupas by feces volume. The Pantaneira breed showed higher attraction to horn flies.
\end{abstract}

Index terms: biological control, flies.

\section{INTRODUÇÃO}

Vários são os fatores envolvidos na atração de insetos hematófagos aos hospedeiros, e entre es-

\footnotetext{
${ }^{1}$ Aceito para publicação em 9 de julho de 1998.

${ }^{2}$ Bióloga, M.Sc., Embrapa-Centro de Pesquisa Agropecuária do Pantanal (CPAP), Caixa Postal 109, CEP 79320-900 Corumbá, MS. Bolsista do CNPq.E-mail: sereno@cpap.embrapa.br

${ }^{3}$ Méd. Veterinário, M.Sc., Embrapa-CPAP.
}

ses se destacam os estímulos: visual, olfativo e térmico (Gillies \& Wilkes, 1969; Vale, 1977; Dalton et al., 1978). Para McLintock \& Depner (1954), os estímulos que atraem a mosca-do-chifre (Haematobia irritans) aos hospedeiros são desconhecidos. Kinzer et al. (1978) apontam a temperatura emitida pela superfície corpórea e o $\mathrm{CO}_{2}$, como fatores que atuam na orientação da H. irritans. Segundo Chamberlain (1981), a proxi- 
midade entre grupos de animais influencia na dispersão de populações da $H$. irritans.

A mosca-do-chifre, introduzida no Brasil por volta de 1980 (Valério \& Guimarães, 1983), foi relatada no Mato Grosso do Sul em 1990 (Honer et al., 1990). No Pantanal, a bovinocultura de corte é explorada extensivamente, em grandes áreas, com predomínio de raças zebuínas (Bos taurus indicus), das quais a Nelore se destaca. Entretanto, ainda pode-se encontrar animais de origem européia, como o bovino Pantaneiro (Bos taurus taurus) (Mazza et al., 1994), habitando as terras alagadas da região. De acordo com os autores, a raça Pantaneira é descendente de raças da Península Ibérica, introduzida no Pantanal há dois séculos, o que lhe proporcionou boa adaptação às condições edafoclimáticas da região.

Os relatos da literatura demonstram que as raças zebuínas possuem maior resistência ao carrapato Boophilus microplus que outras raças bovinas (Hewetson, 1972; Utech et al., 1978). Esta resistência, segundo Hewetson (1972), é um fator hereditário, sendo o Bos indicus mais resistente ao carrapato. Tugwell et al. (1969), em estudo com o gado Brahamn, de origem zebuína, demonstraram que este foi menos atrativo para a mosca-do-chifre do que representantes de cruzamento de gado europeu das raças Aberdeen Angus e Charolês.

Este estudo teve como objetivo comparar o número de pupas de mosca-do-chifre obtido de massas fecais de bovinos das raças Nelore e Pantaneira, e determinar qual delas possui maior atração para a mosca-do-chifre.

\section{MATERIAL E MÉTODOS}

O experimento foi conduzido na Fazenda Nhumirim (latitude $18^{\circ} 59^{\prime} 0^{\prime \prime} \mathrm{S}$, longitude $56^{\circ} 39^{\prime} 0^{\prime \prime} \mathrm{W}$ e altitude $98 \mathrm{~m}$ ), de propriedade da Embrapa-Centro de Pesquisa Agropecuária do Pantanal, localizada na sub-região de Nhecolândia, município de Corumbá, MS. O clima do Pantanal é tropical subúmido (Aw, de Köppen), com uma estação chuvosa (outubro/março) e uma relativamente seca (abril/setembro), segundo Cadavid Garcia (1984). O experimento foi realizado no período de novembro de 1993 a maio de 1994, com coletas mensais de 16 massas fecais de bovinos para cada raça de bovino (Nelore e Pantaneira). A precipitação pluvial, durante os sete meses, foi de $737,3 \mathrm{~mm}$, sendo a máxima no mês de dezembro $(258,1 \mathrm{~mm})$, e a mínima, em novembro $(27,3 \mathrm{~mm})$. As massas fecais frescas eram marcadas em campo, no período da manhã, sendo anotados no ato da marcação, os parâmetros: comprimento, largura, diâmetro e altura. As massas fecais foram marcadas em invernadas distintas para Nelore (537 ha e 100 animais) e para Pantaneira (411 ha e 130 animais). Após sete dias, as massas fecais eram retiradas do campo e colocadas, individualmente, em sacos de linhagem, sendo processada, posteriormente, a coleta de pupas de dípteros, segundo a técnica de Spiller (1966). As pupas recolhidas eram colocadas em vidros cobertos com tecido "voil" e encaminhadas ao laboratório, onde observava-se o nascimento de adultos, o parasitismo e a inviabilidade das pupas. Após 60 dias, as pupas, moscas e parasitóides eram armazenados em vidros com álcool a $70 \%$. O material foi mantido em laboratório, em condições ambientais, à temperatura de $25 \pm 5^{\circ} \mathrm{C}$, umidade relativa de $60 \pm 10 \%$, e fotófase de 12 horas. As espécies de Pteromalidae foram identificadas segundo Boucek (1963). O teste t de Student foi utilizado para análise comparativa dos parâmetros (comprimento, largura, diâmetro e altura) das massas fecais e da abundância de moscas coprófilas no tocante às duas raças. Durante a fase experimental, não foi utilizado nenhum tipo de parasiticida sistêmico na fazenda experimental.

\section{RESULTADOS E DISCUSSÃO}

Das 112 massas fecais de Nelore, 107 (95,5\%) apresentavam pupas de dípteros, e destas, 72 (64,3\%) tinham pupas de $H$. irritans; em Pantaneiro, 105 $(94,0 \%)$ apresentavam pupas, sendo $60(53,6 \%)$ de H. irritans. O número de pupas encontrado nas fezes reflete a infestação de moscas nos animais. As restantes massas fecais $(\mathrm{n}=12)$ não apresentaram pupas de dípteros. Segundo Kunz et al. (1970), o encontro de larvas de dípteros nas massas fecais, independentemente das condições do meio, e as diferenças no número de moscas que emergem das massas fecais podem indicar a existência de diferenças fisiológicas entre os animais, refletindo na susceptibilidade de seu esterco ao desenvolvimento de H. irritans. Entretanto, Sanders \& Dobson (1969) não foram capazes de determinar se a perda de umidade na massa fecal ou de atratividade para a oviposição das fêmeas de dípteros eram responsáveis, ou não, pelo acesso ao substrato.

As massas fecais apresentaram, em média, nas raças Nelore e Pantaneira, respectivamente, $31 \mathrm{~cm}$ 
e $30 \mathrm{~cm}$ de comprimento; $24 \mathrm{~cm}$ e $23 \mathrm{~cm}$ de largura; $93 \mathrm{~cm}$ e $91 \mathrm{~cm}$ de diâmetro; e $7 \mathrm{~cm}$ de altura nas duas. Os resultados deste estudo demonstraram que não houve diferença significativa $(\mathrm{P}>0,05)$ entre os parâmetros comprimento, largura, diâmetro, altura e volume das massas fecais de bovinos das raças Nelore e Pantaneira. Os padrões de massas fecais foram similares, o que demonstra que outros fatores causaram a ausência de dípteros. Estes resultados estão de acordo com os estudos realizados por Sanders \& Dobson (1969), que demonstraram que o tamanho da massa fecal foi um fator que, aparentemente, teve pouco efeito na atratividade para a postura de $H$. irritans. Entretanto, Kunz et al. (1970) observaram que massas fecais depositadas sob as mesmas condições, ao mesmo tempo, apresentavam variação no número de moscas produzidas.

O experimento foi desenvolvido no período de outubro a março, considerado de maior precipitação pluvial no Pantanal e que está relacionado à maior abundância de insetos, estando também, segundo Pott (1994), associado a maior produção de forragens. Segundo Greenham (1972), a Musca vetustissima apresenta um ciclo de produtividade, nas massas fecais, relacionado com o crescimento sazonal da pastagem. Demonstrando esta inter-relação, Kunz (1980) observou que grande número de pupas de $H$. irritans eram produzidas durante o maior crescimento da pastagem. As condições favoráveis interferem na qualidade das pastagens, podendo ter um efeito na microflora ou fauna que influenciam na produção de $H$. irritans, e essa produção parece ser mais dependente do aspecto nutricional das massas fecais do que dos fatores do clima externo.

Foram coletadas 1.715 pupas de dípteros em massas fecais de bovinos Nelore, sendo 237(14\%) delas de $H$. irritans. Nos Pantaneiros, de um total de 2.892 pupas de dípteros, 399 (14\%) pertenciam a $H$. irritans. As pupas provenientes dos dípteros coletadas nos dejetos de bovinos da raça Pantaneira foi maior $(\mathrm{P}<0,05)$ do que em Nelore, sendo estas pertencentes às famílias Muscidae, Sarcophagidae e Sepsidae. Em relação à $H$. irritans, observou-se maior número de pupas $(\mathrm{P}<0,05)$ nas massas fecais de bovinos da raça Pantaneira do que em Nelore.
Nos meses de janeiro e fevereiro de 1994 (Tabela 1), observou-se grande número de H. irritans, e todas as massas fecais de Pantaneiro tinham pupas desta espécie. Nos Pantaneiros foi encontrado maior número total $(\mathrm{P}<0,05)$ de pupas por volume de fezes do que nos Nelores. No entanto, Tugwell et al. (1969) demonstraram que o aumento do grau de sangue da raça Brahman provocou um decréscimo no número de moscas, independentemente da cor do animal, apesar de existirem indicações de efeitos de outros fatores que a porcentagem de sangue desta raça imprime nos cruzamentos. Animais puros Brahman apresentavam menos moscas em comparação a raças como Aberdeen Angus ou Charolês. Entretanto, Tarn et al. (1994) sugerem a utilização de um marcador sorológico na identificação de animais resistentes à mosca-dochifre.

Do total de pupas de $H$. irritans foi obtida emergência de adultos em $69 \%$ e $74 \%$; inviabilidade das pupas em $19 \%$ e $19 \%$; e parasitismo em $12 \%$ e $7 \%$, para pupas recolhidas das massas fecais das raças Nelore e Pantaneira, respectivamente. Em pupas de H. irritans coletadas de Nelore observou-se um total de 28 parasitóides, sendo 27 Spalangia nigroaenea (Pteromalidae) e 1 Trichopria haematobiae (Diapriidae); de Pantaneiros observou-se um total de 27 parasitóides, sendo 22 S. nigroaenea, 4 Spalangia endius e 1 T. haematobiae. Segundo Figg et al. (1983), muitos parasitóides comuns de dípteros que se desenvolvem em massas fecais têm numerosos hospedeiros e são relativamente não seletivos.

$\mathrm{O}$ fato de ter sido observado um número mais elevado de pupas de mosca-do-chifre nas massas fecais de bovinos da raça Pantaneira, pode estar associado à sua origem européia. Apesar da pequena emergência de adultos de mosca-do-chifre nas massas fecais de bovinos da raça Pantaneira durante o período de coletas, o pico que ocorreu nos meses de janeiro e fevereiro/1994 produziu mais adultos de moscas do que o observado na raça Nelore. Os resultados podem sugerir uma maior atratividade da mosca-do-chifre para a raça Pantaneira, durante o período mais favorável para o desenvolvimento das suas larvas nas massas fecais. A menor atratividade de bovinos da raça Nelore à mosca-do-chifre, na 
TABELA 1. Distribuição de Haematobia irritans nas massas fecais de bovinos Nelore e Pantaneiro no período de novembro/93 a maio/94, na Fazenda Nhumirim, Corumbá, MS ${ }^{1}$.

\begin{tabular}{llccccccc}
\hline Raças de bovinos & Pupas coletadas & Nov. & Dez. & Jan. & Fev. & Mar. & Abr. & Maio \\
\hline Nelore & Emergência de adultos & 52 & 33 & 10 & 26 & 9 & 21 & 13 \\
& Pupas inviáveis & 5 & 24 & 1 & 7 & 2 & 2 & 4 \\
& Parasitismo & 2 & 1 & 2 & 9 & 2 & 9 & 3 \\
& Total & $59 \mathrm{a}$ & $58 \mathrm{a}$ & $13 \mathrm{a}$ & $42 \mathrm{a}$ & $13 \mathrm{a}$ & $32 \mathrm{a}$ & $20 \mathrm{a}$ \\
\hline \multirow{2}{*}{ Pantaneira } & Emergência de adultos & 10 & 12 & 158 & 104 & 3 & 4 & 4 \\
& Pupas inviáveis & 4 & 19 & 33 & 18 & 2 & 0 & 1 \\
& Parasitismo & 0 & 5 & 14 & 5 & 0 & 0 & 3 \\
& Total & $14 \mathrm{~b}$ & $36 \mathrm{~b}$ & $205 \mathrm{~b}$ & $127 \mathrm{~b}$ & $5 \mathrm{~b}$ & $4 \mathrm{~b}$ & $8 \mathrm{~b}$ \\
\hline
\end{tabular}

${ }^{1}$ Valores seguidos da mesma letra, na coluna, não diferem entre si $(\mathrm{P}<0,05)$ pelo teste $\mathrm{t}$ de Student.

região, pode ter contribuído para a manutenção destas populações em menor número na região do Pantanal.

\section{CONCLUSÕES}

1. As massas fecais provenientes da raça Pantaneira demonstraram maior atratividade para a mosca-dos-chifres.

2. Na comparação entre as raças estudadas, a massa fecal dos bovinos da raça Pantaneira concorre com melhor potencial como substrato da população de $H$. irritans.

\section{AGRADECIMENTOS}

Ao Dr. Ângelo Prado (UNICAMP), pela identificação das espécies de moscas; ao Dr. Lubomir Masner (Canadá), pela identificação do parasitóide Diapriidae; ao Dr. Ilton Thadeu (ESALQ), pelo auxílio nas análises estatísticas; a Ireno da Silva (CPAP), pelo auxílio nas coletas.

\section{REFERÊNCIAS}

BOUCEK, Z. A taxonomic study in Spalangia Latr. (Hymenoptera, Chalcidoidea). Acta Entomologica Musei Naturae Pragae, v.35, p.429-511, 1963.

CADAVID GARCIA, E.A. O clima no Pantanal Matogrossense, Corumbá. Corumbá: Embrapa-CPAP, 1984. 39p. (Embrapa-CPAP. Circular técnica, 14).
CHAMBERLAIN, W.F. Dispersal of horn flies: effect of host proximity. Southwestern Entomologist, v.6, n.4, p.316-325, 1981.

DALTON, L.W.; KINZER, H.G.; REEVES, J.M.; ATMAR, J.W. Host location in the horn fly: roles of heat, $\mathrm{CO}_{2}$, water vapor and cow-produced odors in attraction. Southwestern Entomologist, v.3, p.147153,1978 .

FIGG, D.E.; HALL, R.D.; THOMAS, G.D. Insect parasites associated with Diptera developing in bovine dung pats on central Missouri pastures. Environmental Entomology, v.12, n.3, p.961-966, 1983.

GILLIES, M.T.; WILKES, T.J. A comparison of the range of attraction of animal baits and of carbon dioxide for some West African mosquitoes. Bulletin of Entomological Research, v.59, p.441-456, 1969.

GREENHAM, P.M. The effects of the variability of cattle dung on the multiplication of the bushfly (Musca vetustissima Walk.). Journal of Animal Ecology, v.41, n.1, p.153-165, 1972.

HEWETSON, R.W. The inheritance of resistance by cattle to cattle tick. Australian Veterinary Journal, v.48, p.299-303, 1972.

HONER, M.R.; BIANCHIN, I.; GOMES, A. Mosca-doschifres: histórico, biologia e controle. Campo Grande: Embrapa-CNPGC, 1990. 34p. (EmbrapaCNPGC. Documentos, 45).

KINZER, H.G.; REEVES, J.M.; ATMAR, J.W. Host location by the horn fly: field evaluation of an artificial device for measuring attraction to various stimuli. 
Environmental Entomology, v.7, n.3, p.375-378, 1978.

KUNZ, S.E. Horn fly production as affected by seasonal changes in rangeland forage conditions. Southwestern Entomologist, v.5, n.2, p.80-83, 1980.

KUNZ, S.E.; BLUME, R.R.; HOGAN, B.F.; MATTER, J.J. Biological and ecological investigations of horn flies in central Texas: influence of time of manure deposition on oviposition. Journal of Economic Entomology, v.63, n.3, p.930-933, 1970.

MAZZA, M.C.M.; MAZZA, C.A.S.; SERENO, J.R.B.; SANTOS, S.A.; PELLEGRIN, A.O. Etnobiologia e conservação do bovino Pantaneiro. Corumbá: Embrapa-CPAP/Brasília: Embrapa-SPI, 1994. 61p.

McLINTOCK, J.; DEPNER, K.R. A review of the lifehistory and habits of horn fly, Siphona irritans (L.) (Diptera:Muscidae). Canadian Entomologist, v.86, p.20-33, 1954.

POTT, A. Ecossistema Pantanal. In: REUNIÓN SOBRE UTILIZACIÓN Y MANEJO DE PASTIZALES. Montevideo: Juan Puignan, 1994. p.18-23.

SANDERS, D.P.; DOBSON, R.C. Contributions to the biology of the horn fly. Journal of Economic Entomology, v.62, n.6, p.1362-1366, 1969.
SPILLER, D. House flies. In: SMITH, C.N. (Ed.). Insect colonization and mass production. New York: Academic, 1966. p.203-225.

TARN, C.Y.; ROSENKRANS JUNIOR, C.F.; STEELMAN, C.D.; BROWN JUNIOR, A.H.; JOHNSON, Z.B. Plasma characteristics of beef cattle classified as resistant or susceptible to horn flies. Journal of Animal Science, v.72, n.4, p.886-890, 1994.

TUGWELL, P.; BURNS, E.C.; TURNER, J.W. Brahman breeding as a factor affecting the attractiveness or repellency of cattle to the horn fly. Journal of Economic Entomology, v.62, n.1, p.56-57, 1969.

UTECH, K.B.; WHARTON, R.H.; KERR, J.D. Resistance to Boophilus microplus (Canestrini) in different breeds of cattle. Australian Journal of Agricultural Research, v.29, p.885-895, 1978.

VALE, G.A. The flight of tsetse flies (Diptera: Glossinidae) to and from a stationary ox. Bulletin of Entomology Research, v.67, p.297-303, 1977.

VALÉRIO, J.R.; GUIMARÃES, J.R. Sobre a ocorrência de uma nova praga, Haematobia irritans (L.) (Diptera, Muscidae) no Brasil. Revista Brasileira de Zoologia, São Paulo, v.1, n.4, p.417-418, 1983. 